\title{
Qualidade e segurança no uso da insulinoterapia pela população idosa
}

Quality and safety in the use of insulin therapy by the aged population

Calidad y seguridad en el uso de la insulinoterapia por parte de la población de edad avanzada

\section{Michele Rohde Krolow ${ }^{\mathrm{I}}$, Karla Pereira MachadoII, Adriéli Timm Oliveira ${ }^{\mathrm{III}}$, Mariangela Uhlmann Soares ${ }^{\mathrm{IV}}$, Pâmela Moraes Volz ${ }^{\mathrm{v}}$, Elaine Thumévi}

\begin{abstract}
Resumo: Objetivo: avaliar a qualidade e segurança individual e coletiva no uso de insulina pela população idosa em um município de grande porte do sul do Brasil. Método: estudo transversal de base populacional realizado em 2016-2017 com idosos de 68 anos ou mais. As entrevistas foram realizadas nos domicílios dos idosos. O desfecho foi medido pela forma de utilização e descarte das seringas e agulhas. Realizou-se análise descritiva mediante cálculo das proporções e projeções dos dados para os idosos do município. Resultados: foram entrevistados 735 idosos. A prevalência de diabetes foi de $20,0 \%$, e $13,8 \%$ faziam uso de insulina injetável. A reutilização das seringas e agulhas foi relatada por $55,0 \%$ e $65,0 \%$ referiram descartar o material direto no lixo comum. Conclusões: a qualidade e a segurança dos idosos em insulinoterapia estão comprometidas, sendo necessário implementar estratégias de educação em saúde que visem melhorar o conhecimento e acesso as orientações adequadas.
\end{abstract}

Descritores: Idoso; Diabetes Mellitus; Insulina; Equipamentos Descartáveis; Segurança do Paciente

Abstract: Objective: to evaluate individual and collective quality and safety in the use of insulin by the aged population in a large city of southern Brazil. Method: a cross-sectional population-based study conducted in 20162017 with older adults aged 68 and over. The interviews were conducted in the older adults' homes. The outcome

\footnotetext{
${ }^{\text {I }}$ Mestranda em Ciências no programa de pós-graduação em Enfermagem, Universidade Federal de Pelotas, Pelotas, RS, Brasil. E-mail: michelemrk@hotmail.com, Orcid: 0000-0002-7518-7039.

II Mestre em Saúde Pública pela Universidade Federal do Rio Grande, Doutoranda em Ciências no Programa de Pós-Graduação em Enfermagem na Universidade Federal de Pelotas, Pelotas, RS, Brasil. E-mail: karlamachadok@gmail.com, Orcid: 0000-0003-1765-1435.

III Mestranda em Ciências no programa de pós-graduação em Enfermagem, Universidade Federal de Pelotas, Pelotas, RS, Brasil. E-mail: adrielioliveira85@gmail.com, Orcid: 0000-0002-1174-3569.

${ }^{\text {IV }}$ Doutora em Ciências, Universidade Federal de Pelotas, Pelotas, RS, Brasil. E-mail: mariangela.soares@gmail.com, Orcid: 0000-0002-6483-4931.

v Docente na Faculdade de Medicina - FURG. Doutora em Ciências, Universidade Federal de Pelotas, Pelotas, RS, Brasil. E-mail: pammi.volz@gmail.com, Orcid: orcid.org/0000-0002-8548-7190.

${ }^{V I}$ Docente no Programa de Pós-graduação em Enfermagem - UFPel. Doutora em Epidemiologia. Universidade Federal de Pelotas. Pelotas, RS, Brasil. E-mail: elainethume@gmail.com, Orcid: 0000-0002-1169-8884.
}

* Extraído do trabalho de conclusão de curso "Qualidade e segurança no uso da insulinoterapia pela população idosa de Bagé, RS”, Graduação em enfermagem, Universidade Federal de Pelotas, ano 2019. 
was measured by the way in which syringes and needles were used and disposed of. Descriptive analysis was performed by calculating the data proportions and projections for the older adults in the municipality. Results: 735 older adults were interviewed. The prevalence of diabetes was $20.0 \%$, and $13.8 \%$ made use of injectable insulin. Syringes and needle reuse was reported by $55.0 \%$, and $65.0 \%$ mentioned discarding the material directly in regular garbage. Conclusions: the quality and safety of the older adults in insulin therapy are compromised, and it is necessary to implement health education strategies aimed at improving knowledge and access to the appropriate guidelines.

Descriptors: Aged; Diabetes Mellitus; Insulin; Disposable Equipment; Patient Safety

Resumo: Objetivo: evaluar la calidad y la seguridad individual y colectiva en el uso de insulina por parte de la población de edad avanzada en un importante municipio del sur de Brasil. Método: estudio transversal de base poblacional realizado en 2016-2017 con ancianos de al menos 68 años de edad. Las entrevistas se realizaron en los domicilios de las personas de edad avanzada. El resultado se midió considerando la forma de utilización y descarte de las jeringas y agujas. Se realizó un análisis descriptivo mediante el cálculo de las proporciones y proyecciones de los datos para los ancianos del municipio. Resultados: se entrevistó a un total de 735 personas de edad avanzada. La prevalencia de diabetes fue del 20,0\%, y el 13,8\% utilizaba insulina inyectable. El 55,0\% indicó que reutilizaba las jeringas y agujas y el 65,0\% mencionó que desechaba el material directamente en la basura común. Conclusiones: la calidad y la seguridad de las personas de edad avanzada sometidas a insulinoterapia están en riesgo, por lo que resulta necesario implementar estrategias de educación en salud que tengan por objetivo mejorar el conocimiento y el acceso a las pautas adecuadas.

Descriptores: Anciano; Diabetes Mellitus; Insulina; Equipos Desechables; Seguridad del Paciente

\section{Introdução}

Mundialmente, estima-se que 415 milhões de pessoas tenham diagnóstico médico de Diabetes Mellitus (DM). ${ }^{1}$ Dados da Federação Internacional de Diabetes em 220 países, constataram que a prevalência de DM tipo 2 no ano 2015 variou de 4,3\% na África, 7,0\% na Europa, 9,1\% na América Central e do Sul, à 12,7\% no Oriente médio e em nível mundial a prevalência foi de $8,2 \% .^{2}$ No Brasil atualmente há $6,9 \%$ da população com a doença, o que representa mais de 13 milhões de pessoas. ${ }^{1}$

As condições genéticas e comportamentais são os fatores de risco mais comuns para a ocorrência de diabetes, sendo a doença caracterizada pela elevação nos níveis glicêmicos que ocorrem pela deficiência na produção da insulina ou na sua ação no organismo. ${ }^{3-4}$ No controle dos níveis de glicose pode ocorrer hiperglicemia ou hipoglicemia e muitos indivíduos com DM fazem uso de medicamentos orais ou injetáveis para regular os valores glicêmicos no organismo, como a insulina. ${ }^{1,4}$ 
3 | Krolow MR, Machado KP, Oliveira AT, Soares MU, Volz PM, Thumé E

A reutilização das agulhas na aplicação da insulina é uma preocupação devido a possibilidade de causar dor, sangramento e aumento no risco de contaminação. ${ }^{1-2}$ Quando usada diariamente exige alguns cuidados básicos para garantir a segurança e a qualidade na aplicação. As recomendações ainda são divergentes e não há consenso entre o Ministério da Saúde (MS) e a Sociedade Brasileira de Diabetes (SBD). O primeiro orienta a utilização das seringas e agulhas descartáveis por até oito vezes pela mesma pessoa, entretanto, a SBD recomenda que a última, seja de uso único e descartadas logo após o uso. ${ }^{1,4}$

O descarte das agulhas e seringas deve ser realizado em recipientes específicos para materiais perfurocortantes ou frascos plásticos rígidos de boca larga e, descartados como material contaminante pelos serviços de saúde. ${ }^{5} \mathrm{Na}$ maioria das vezes, o descarte nos domicílios é realizado no lixo doméstico e em recipientes inadequados causando risco biológico, domiciliar e ambiental, além da possibilidade de causar acidentes no manuseio destes por catadores ou trabalhadores da coleta urbana. ${ }^{6}$

$\mathrm{Na}$ África Oriental, um estudo realizado com informações da EADSG (East African Diabetes Study Group) orienta que as seringas e agulhas sejam utilizadas apenas uma vez, mas se for reutilizada, que não seja por mais de cinco vezes. O descarte seguro deve ser orientado pelos profissionais de saúde e é responsabilidade do paciente a separação em caixas de coleta, sendo à prova de perfuração. Após, a destinação do material deve ser dos próprios fabricantes para seus devidos fins. $^{7}$

A segurança do paciente quanto ao uso da insulina, se caracteriza por um conjunto de ações para evitar, prevenir e minimizar problemas decorrentes dos cuidados e intervenções em saúde. ${ }^{5}$ Os usuários insulinodependentes realizam a aplicação diária de insulina, para isto, precisam de orientações que incluem a boa técnica da aplicação injetável e as formas de acondicionamento e descarte do material de modo a garantir o uso de forma segura. 
Durante muitos anos, à temática referente a reutilização de seringas e agulhas e o descarte do material foi considerada um problema de saúde e se mantém atual e relevante. ${ }^{1}$ Portanto, este estudo responde a seguinte questão: "Como pode ser avaliada a qualidade e a segurança no uso da insulina pela população idosa de Bagé?”. Nessa direção, o objetivo é avaliar a qualidade e a segurança individual e coletiva no uso de insulina pela população idosa em um município de grande porte do sul do Brasil.

\section{Método}

Trata-se de um estudo transversal de base populacional, em área de abrangência dos serviços de atenção primária à saúde. Este artigo utilizou dados coletados no acompanhamento do estudo de coorte intitulado "Saúde do Idoso Gaúcho de Bagé, RS (SIGa-Bagé)", que teve por objetivo identificar as modificações na situação de saúde dos idosos e as contribuições da Estratégia Saúde da Família (ESF) no atendimento às necessidades em saúde da área urbana de Bagé no período de 2008 a 2016/2017.

O município de Bagé está situado no sul do estado do Rio Grande do Sul (RS) a 393 km da capital do estado, Porto Alegre. A população estimada no município na época da coleta de dados (2017) era de 122.209 habitantes, o Índice de Desenvolvimento Humano (IDH) era de 0,740 e a expectativa de vida ao nascer era em média de 75,9 anos. ${ }^{8}$ O sistema municipal de saúde em 2017, era formado por 19 Unidades Básicas de Saúde (UBS) com ESFs e 3 unidades tradicionais localizadas na zona urbana do município.9

A amostra é constituída por idosos com 68 anos ou mais de idade, entrevistados no estudo de linha de base da constituição da coorte em 2008, no qual o critério de inclusão era ter, no mínimo, 60 anos. Foram excluídos aqueles idosos que, durante a coleta de dados, estavam institucionalizados, haviam mudado de município ou estavam viajando. 
5 | Krolow MR, Machado KP, Oliveira AT, Soares MU, Volz PM, Thumé E

O tamanho de amostra em 2008, foi calculado considerando $10 \%$ de perdas e recusas, um efeito de delineamento de $1.3,80 \%$ de poder para detectar riscos relativos de 1.5 exposições que afetavam no mínimo, $4 \%$ da população. O processo de amostragem se deu por múltiplos estágios. Inicialmente, a amostra foi localizada a partir da área de abrangência de cada uma das UBS e, posteriormente, dividida em microáreas, com identificação numérica de cada quadra, sorteando aleatoriamente o ponto inicial de coleta dos dados. Com o intuito de garantir que todos os domicílios tivessem a mesma probabilidade de compor a amostra e garantir a dispersão desta no território, foi empregado o pulo sistemático de uma a cada cinco residências. ${ }^{10}$

Este estudo utiliza a coleta de dados do acompanhamento da coorte que ocorreu de setembro de 2016 a agosto de 2017, a fim de contatar os idosos entrevistados em 2008. A coleta de dados foi realizada por entrevistadores previamente capacitados e treinados sendo realizadas no domicílio do próprio idoso. Os entrevistadores tinham uma lista com o nome, telefone, endereço e UBS de referência dos idosos, com o propósito de contatá-los. Foi utilizado questionário eletrônico estruturado com questões pré-codificadas, padronizado, previamente testado e aplicado com o uso de aparelho digital Personal Digital Assistent (PDA). O instrumento completo foi composto por 757 perguntas abertas e fechadas, além de realização de medidas antropométricas.

Antes da coleta de dados em 2016/2017, foi realizado um estudo piloto com idosos de uma instituição de longa permanência do município de Bagé, a fim de testar os instrumentos, o manual de instruções, a organização do trabalho de campo e o desempenho dos entrevistadores. A logística previu três tentativas de localização do idoso e aplicação do questionário, caso contrário era considerado perda/recusa, não sendo admitidas substituições.

Para avaliar o desfecho - qualidade e segurança no processo de uso de insulina - foram utilizadas as questões: $\mathrm{O}(\mathrm{A})$ sr.(a) utiliza novamente a seringa? (não/sim) Se sim, quantas vezes?, Como o(a) sr.(a) descarta (joga fora) a seringa? (direto no lixo comum/coloca a agulha e a seringa em um recipiente rígido e coloca no lixo comum/coloca a agulha e a seringa em recipiente rígido 
Qualidade e segurança no uso da insulinoterapia pela população idosa | 6

e leva para a UBS/coloca a agulha e a seringa em recipiente rígido e entrega para agente comunitário de saúde (ACS) ou outro profissional que realiza a visita domiciliar/outro, qual?.

Os idosos insulinodependentes foram identificados por meio das questões: Em algum momento da sua vida algum médico disse que o(a) sr.(a) tem Diabetes, (açúcar no sangue), mesmo que controlada? (não/sim). Para os idosos que responderam "sim", questionou-se: $\mathrm{O}(\mathrm{A})$ sr.(a) usa insulina injetável? (não/sim), Quem faz a aplicação da insulina no(a) sr.(a)? (o próprio idoso/algum familiar/profissional de saúde/outro, qual?.

Para fins de análise, foi considerada qualidade no uso de insulina quando os idosos utilizaram apenas uma vez a seringa e a agulha de aplicação, não reutilizando o material conforme orientação da SBD e da Agência Nacional de Vigilância Sanitária (ANVISA) na resolução (RE) nº 2.605,29 de 11 de agosto de 2006. ${ }^{1,11}$ Quanto ao armazenamento e descarte, foi considerado seguro o processo quando armazenado os perfurocortantes em material rígido e entregue na UBS, levado até o serviço ou entregue a algum profissional em visita domiciliar conforme Resolução da Diretoria Colegiada (RDC) № 306, de 7 de dezembro de $2004 .{ }^{12}$

Para compor a análise descritiva, foram utilizadas as seguintes variáveis demográficas, socioeconômicas, morbidades e situação de saúde: sexo (masculino/feminino), idade: (68 - 79 anos/ $\geq 80$ anos), cor da pele (branca/preta, amarela, parda, indígena), escolaridade (nenhuma/1 a 7 anos/ $\geq 8$ anos); classificação socioeconômica conforme critérios da Associação Brasileira de Empresas de Pesquisa (ABEP) (A e B/C/D e E), ${ }^{13}$ aposentadoria (não/sim), situação conjugal (com companheiro ou casado; sem companheiro; solteiro/viúvo), mora sozinho (não/sim), autopercepção de saúde (péssima e ruim/regular/ótima e boa), plano de saúde (não/sim) e tipo de UBS de cobertura (Tradicional/ESF).

A análise dos dados foi realizada no programa estatístico Stata 12.0 (StataCorp/College, Estados Unidos da América). Na análise foi utilizada a estatística descritiva, com cálculo das proporções e seus respectivos intervalos de confiança (IC95\%). Após, foram calculados os valores 
7 | Krolow MR, Machado KP, Oliveira AT, Soares MU, Volz PM, Thumé E

através de projeção por meio do Microsoft $E_{X c e}{ }^{\circledR}$, para a população idosa do município de Bagé acima de 65 anos, utilizando dados da Fundação de Economia e Estatística (2017). ${ }^{14}$

A pesquisa foi conduzida de acordo com os padrões éticos exigidos (Resoluções 466/2012 510/2016 - 580/2018, do MS e o estudo foi submetido à Plataforma Brasil, avaliado pelo Comitê de Ética em Pesquisa da Faculdade de Medicina da Universidade Federal de Pelotas e aprovado sob parecer 678.664 em 29 de maio de 2014.

\section{Resultados}

Em 2016-2017 foram entrevistados 735 idosos. Dos idosos que não foram entrevistados no segundo acompanhamento, 81 (5,1\%) foram recusas e $220(13,8 \%)$ foram perdas (incluindo não localizados, institucionalizados, mudaram-se para outro município e perdas na transferência de dados). A amostra foi constituída, majoritariamente por mulheres $(65,4 \% ; n=481)$ e idosos com cor de pele branca $(82,2 \% ; n=604)$. A idade média foi de 77,2 anos ( $\mathrm{DP} \pm 6,2$ anos). Quanto à classificação socioeconômica (ABEP), 46,1\% (n=332) encontrava-se nos estratos D/E. A autopercepção de saúde ótima/boa foi relatada por $53,7 \%(\mathrm{n}=382)$ da amostra e $54,4 \%(\mathrm{n}=400)$ dos idosos estavam cobertos pela ESF (dados não apresentados em tabela).

$\mathrm{Na}$ Tabela 1, são apresentadas as frequências relativas (\%) dos idosos com diagnóstico médico de DM e em uso de insulina. Observa-se que metade eram do sexo masculino ( $\mathrm{n}=10)$, três quartos $(n=15)$ apresentavam idades entre 68 e 79 anos, a maioria era de cor de pele $(n=17)$ branca e possuía entre 1 e 7 anos de escolaridade ( $n=14)$. Metade dos idosos $(n=10)$ eram da classe socioeconômica $\mathrm{D} / \mathrm{E}$, a maioria estava aposentada $(\mathrm{n}=18)$ e não moravam sozinhos $(\mathrm{n}=14)$, e ainda, a maior parte deles moravam em área de cobertura da ESF ( $\mathrm{n}=14)$ (Tabela 1). 
Qualidade e segurança no uso da insulinoterapia pela população idosa $\mid 8$

Tabela 1 - Descrição da amostra de acordo com as características demográficas e socioeconômicas dos idosos insulinodependentes. Bagé/RS, 2016/17 ( $\mathrm{n}=20$ ).

\begin{tabular}{llll}
\hline & Variável & N & \% \\
\hline Sexo & Masculino & 10 & \\
& Feminino & 10 & 50,0 \\
& & & 50,0
\end{tabular}

Idade

$\begin{array}{lcc}68-79 \text { anos } & 15 & 75,0 \\ \geq 80 \text { anos } & 5 & 25,0\end{array}$

\section{Cor da pele}

$\begin{array}{lcc}\text { Branca } & 17 & 85,0 \\ \text { Preta, amarela, parda, indígena } & 3 & 15,0\end{array}$

\section{Escolaridade}

$\begin{array}{lcc}\text { Nenhuma } & 2 & 10,0 \\ 1 \text { a } 7 \text { anos } & 14 & 70,0 \\ \geq 8 \text { anos } & 4 & 20,0 \\ \text { ificação socioeconômica } & & \\ \text { A/B } & 2 & 10,0 \\ \text { C } & 8 & 40,0 \\ \text { D/E } & 10 & 50,0\end{array}$

Aposentadoria

Não $\quad 2 \quad 10,0$

Sim $18 \quad 90,0$

Situação conjugal

Com companheiro/casado $\quad 8 \quad 40,0$

Sem companheiro/solteiro $\quad 5 \quad 25,0$

$\begin{array}{lll}\text { Viúvo } & 7 & 35,0\end{array}$

Mora sozinho

$\begin{array}{lcc}\text { Não } & 14 & 70,0 \\ \text { Sim } & 6 & 30,0\end{array}$

Autopercepção de saúde

Péssima/ruim $\quad 1 \quad 5,2$

Regular $\quad 15 \quad 79,0$

Ótima/boa $\quad 3 \quad 15,8$

Plano de Saúde

$\begin{array}{lcc}\text { Não } & 13 & 65,0 \\ \text { Sim } & 7 & 35,0\end{array}$

Tipo da UBS de cobertura

$\begin{array}{lll}\text { Tradicional } & 6 & 30,0\end{array}$

ESF $\quad 14 \quad 70,0$

$\mathrm{N}=$ frequência absoluta; \%=frequência relativa; UBS: Unidade Básica de Saúde; ESF: Estratégia Saúde da Família. 
9 | Krolow MR, Machado KP, Oliveira AT, Soares MU, Volz PM, Thumé E

A prevalência de DM foi de 20,0\% (IC95\%:17,1; 22,9), o uso de insulina injetável foi referido por $13,8 \%$ dos idosos (IC95\%: 8,1; 19,5), quando questionado quem aplicava a insulina, 65,0\% (IC95\%: $42,1 ; 87,9)$ referiu que era o próprio idoso e $25,0 \%$ (IC95\%: 4,2; 45,8) algum familiar (Tabela 2).

A reutilização da seringa e agulha foi relatada por $55,0 \%$ dos idosos que faziam uso de insulina injetável e reutilizavam o equipamento duas vezes ou mais. Em relação ao descarte do material perfurocortante, $65,0 \%$ descartavam diretamente no lixo comum, $15,0 \%$ realizavam a queima do material, e 10,0\% colocavam em recipiente rígido e descartavam no lixo comum e colocavam em recipiente rígido e levavam para a UBS ou entregavam para algum profissional de saúde (Tabela 2).

Ainda, na tabela 2 é apresentada a projeção dos desfechos em estudo com base na população estimada de 14.586 idosos em Bagé acima de 65 anos. ${ }^{14}$ Utilizando a prevalência da amostra, o total estimado de idosos com diagnóstico de DM no município seria de 2.917 indivíduos. Extrapolando os percentuais deste estudo, cerca de 397 idosos usariam insulina para o controle de diabetes e aproximadamente 258 realizariam autoaplicação. A reutilização do material projetada seria realizada por 218 idosos, dos quais 179 idosos utilizariam por duas vezes e 40 idosos por três vezes. Quanto ao descarte do material, um total de 258 idosos descartariam seringas e agulhas diretamente na lixeira comum.

Tabela 2 - Prevalência das variáveis de desfecho do estudo SIGa-Bagé 2016/17 e extrapolação para a população de idosos residentes na zona urbana do município de Bagé. 2016/17 (n=735).

\begin{tabular}{|c|c|c|c|}
\hline \multirow{3}{*}{ Variáveis } & \multicolumn{2}{|c|}{$\begin{array}{c}\text { Dados SIGa-Bagé } \\
2016 / 17\end{array}$} & \multirow{3}{*}{$\begin{array}{c}\begin{array}{c}\text { Projeções para idosos }>65 \\
\text { anos de Bagé, RS }\end{array} \\
\mathrm{N}\end{array}$} \\
\hline & & & \\
\hline & $\mathrm{n}$ & $\%$ & \\
\hline \multicolumn{4}{|l|}{ Diabetes $(\mathrm{n}=734)$} \\
\hline $\operatorname{Sim}$ & 147 & 20,0 & 2.917 \\
\hline Não & 587 & 80,0 & 11.669 \\
\hline \multicolumn{4}{|l|}{ Uso de Insulina $(n=147)$} \\
\hline Sim & 20 & 13,6 & 397 \\
\hline Não & 127 & 86,4 & 2521 \\
\hline \multicolumn{4}{|l|}{ Aplicação (n=20) } \\
\hline Próprio idoso & 13 & 65,0 & 258 \\
\hline Familiar & 5 & 25,0 & 99 \\
\hline
\end{tabular}




\begin{tabular}{lccc}
\hline Outros & 2 & 10,0 & 40 \\
Reutilização $(\mathrm{n}=20)$ & & & \\
$\quad$ Sim & 11 & 55,0 & 218 \\
$\quad$ Não & 9 & 45,0 & 179 \\
Quantidade de vezes (n=11) & & & 179 \\
$\quad$ vezes & 9 & 81,8 & 40 \\
3 vezes & 2 & 18,2 & \\
Descarte (n=20) & & & 258 \\
$\quad$ Diretamente no lixo comum & 13 & 65,0 & 60 \\
$\quad$ Queima & 3 & 15,0 & 40 \\
$\quad$ Recipiente rígido lixo comum & 2 & 10,0 & 40 \\
$\quad$ Recipiente rígido na UBS & 2 & 10,0 & $\mathbf{1 4 . 5 8 6 ^ { * }}$ \\
Total & $\mathbf{7 3 5}$ & $\mathbf{1 0 0 , 0}$ & \\
\hline
\end{tabular}

$\mathrm{N}=$ frequência absoluta; \%=frequência relativa; SIGa-Bagé... *Número de idosos em Bagé de acordo com Fundação de Economia e Estatística, 2017. ${ }^{12}$

\section{Discussão}

Entre os principais resultados, destaca-se que a reutilização da agulha e da seringa e o descarte do material perfurocortante de maneira errada, comprometem a qualidade no processo e a segurança da aplicação e do descarte relacionados ao uso da insulina.

No presente estudo, verificou-se que um quinto dos idosos possuíam o diagnóstico de DM. Observando que no período de 2001 a 2017 a prevalência variou entre 6,2\% e 22,4\% no Brasil. ${ }^{15-18}$ De acordo com dados do Sistema de Informação sobre Mortalidade (SIM) em 2016 no município de Bagé, as doenças cardiovasculares corresponderam 34\% dos óbitos, sendo a DM responsável por 9\% destes. Ainda, 5092 pacientes foram cadastrados no Sistema de Informação da Atenção Básica com diagnóstico de DM no ano. ${ }^{8}$

Verifica-se que, apesar dos anos, o número de pacientes com diagnostico de diabetes segue aumentando, além dos óbitos causados pela própria doença. E mesmo com as orientações e fornecimento de informações de práticas diárias para o controle do açúcar, como cuidados na alimentação e a prática de atividade física, há indivíduos que não conseguem colocá-las em ação, não obtendo uma mudança em seus hábitos de vida. É necessário além do repasse das 
11 | Krolow MR, Machado KP, Oliveira AT, Soares MU, Volz PM, Thumé E

orientações, que os profissionais de saúde estejam atentos a outros motivos que levam essas pessoas a não realizarem essas mudanças consideradas simples e efetivas, como os fatores ambientais, econômicos e sociais. ${ }^{4,19}$

Em São Paulo, nos anos de 2001 e 2002 foram entrevistados 1949 idosos, sendo 19\% usuários de insulina. ${ }^{16}$ Em um estudo comparativo com dados do sistema de Vigilância de Fatores de Risco e Proteção para Doenças Crônicas por Inquérito Telefônico (Vigitel) as prevalências de uso da insulina em 2012 e 2016 foram de 17,6\% e 19,7\% respectivamente. ${ }^{20}$ Conforme os estudos encontrados na literatura, ${ }^{15-18}$ a prevalência do uso de insulinoterapia não sofreu variação com os anos e tem se mantido estável, da mesma forma que a prevalência da doença. Em Bagé, aproximadamente um em cada sete idosos fazia uso de insulina injetável. As prevalências reforçam a necessidade dos profissionais de saúde e rede de apoio informal estarem atentos para a prática de aplicação da insulina, para seguir a prescrição corretamente, ao aplicar a dose diária e atentar aos cuidados de assepsia do local e higiene das mãos.

Quanto a reutilização da agulha e seringa para aplicação, foi observado que mais da metade dos idosos deste estudo faziam o reuso do material, o que compromete a qualidade da atenção. Em Cajazeiras (Paraíba) em 2015, estudo realizado com 15 usuários de insulina entre 10 e 85 anos, verificou que $80 \%$ reutilizavam agulhas e seringas, variando de 2 até 15 aplicações com o mesmo material, $50 \%$ dos usuários justificaram o reuso pelo não fornecimento do material pelo Sistema Único de Saúde (SUS), e os demais referiram a condição financeira e/ou economia do material. ${ }^{21}$ Outro estudo realizado em Porto Alegre (Rio Grande do Sul) em 2017, com 168 diabéticos insulinodependentes e familiares responsáveis pela aplicação da insulina, a reutilização do material perfurocortante foi referida por $93,4 \%$ dos indivíduos, sendo que alguns mencionaram reutilizar por até 30 dias. ${ }^{22}$ 
A SBD, ${ }^{1}$ a Organização Mundial da $S_{a u ́ d e}{ }^{23}$ e os próprios fabricantes identificam agulhas e seringas como material de uso único, o que diverge da recomendação do $\mathrm{MS},{ }^{4}$ que autoriza a reutilização desses materiais desde que com os devidos cuidados.

No município de Bagé os profissionais de saúde das UBS seguem as diretrizes do MS para orientar os usuários com diabetes em terapêutica insulínica. Cabe ressaltar que o material descartável não é disponibilizado integralmente na rede pública de saúde, dificultando o acesso da população que diariamente realiza a aplicação do medicamento.

Alguns pacientes optam pela reutilização das agulhas de insulina por conveniência, economia, falta de insumo e preocupação ambiental. O reaproveitamento da agulha causa alterações como: perda da lubrificação e da afiação, alterações no bísel, risco de quebra da agulha durante a aplicação e bloqueio do fluxo devido à cristalização da insulina. ${ }^{1}$

Os idosos deste estudo reutilizaram por menos tempo as agulhas e seringas em comparação com os demais estudos com usuários de insulina, embora esteja aquém do recomendado. ${ }^{21-22}$ Observa-se que as UBS do município de Bagé em sua maioria são ESF o que acaba beneficiando o vínculo com os usuários, e pode indicar um maior fornecimento de orientações pelos profissionais de saúde de forma acessível para a compreensão da população alvo.

No estudo já citado acima, foi realizada análise bacteriológica em 20 agulhas de perfurocortantes reutilizadas e os autores identificaram que, após 24 horas de incubação do material, 45\% das agulhas apresentaram crescimento bacteriano constatando a contaminação, e, do público estudado, 46,7\% referiram não realizar cuidado nenhum de higiene incluindo a lavagem das mãos para aplicação. E destaca-se que $60 \%$ dos idosos referiram alguma reação no local de aplicação, mencionando sangramento, eritema, hematoma, edema e lipodistrofia. ${ }^{21}$

Portanto, a contaminação de materiais perfurocortantes compromete a qualidade e a segurança no uso da insulina e exige orientações sobre cuidados de higienização e investimento na oferta de material em quantidade suficiente. Os riscos dessa contaminação são grandes, dependendo 
13 | Krolow MR, Machado KP, Oliveira AT, Soares MU, Volz PM, Thumé E

da bactéria colonizada, pode causar abscessos e evasão pela corrente sanguínea, podendo ocasionar a morte de algumas células, infecção local ou sistêmica e consequentemente à internação. ${ }^{21}$

Quanto ao descarte do material perfurocortante no domicílio, marcador de segurança neste estudo, observou-se que, quase a totalidade da população não descartava de modo correto em recipiente adequado e retorno à UBS para destinação adequada.

No estudo desenvolvido no ambulatório de endocrinologia de um hospital escola de Campinas (São Paulo), com 70 indivíduos insulinodependentes, 34,3\% relataram colocar em um recipiente e levar para a UBS, 51,4\% dos indivíduos relataram descartar o material no lixo doméstico, e 14,3\% realizavam a queima do lixo, ainda foi questionado quanto ao conhecimento sobre lixo doméstico e biológico, e apenas 5,7\% dos entrevistados sabiam a diferença. ${ }^{24}$ Em Fortaleza (Ceará), um estudo selecionou 143 usuários de Insulinoterapia entre 2014 e 2015 e constatou que $57,1 \%$ dos entrevistados descartavam os materiais perfurocortantes no lixo comum e $48,6 \%$ dos usuários relataram receber orientações durante as consultas sobre o correto descarte, sendo em $90,0 \%$ dos casos, o enfermeiro quem realizava essa orientação. Ainda, 2,9\% dos entrevistados relataram já ter ocorrido acidentes com os moradores do domicílio devido o descarte indevido do material. ${ }^{25}$

Em decorrência do potencial degradante, poluente contra o meio ambiente e infeccioso contra a saúde da população que pode vir a ter contato com esses materiais, os resíduos de serviços de saúde, como os materiais perfurocortantes, exigem cuidados especiais e técnicas corretas de manejo e gerenciamento. O descarte incorreto pode levar também a proliferação de insetos vetores, a contaminação direta dos profissionais que manuseiam o lixo, além de tragédias ambientais, principalmente em períodos de chuvas fortes. ${ }^{26}$

A preocupação com o meio ambiente é observada desde 1992 com o lançamento da Agenda 21, um plano de ação para a construção de uma sociedade sustentável unindo métodos de proteção ambiental, justiça social e eficiência econômica, assinado por 179 países durante a 
Conferência das Nações Unidas sobre Meio Ambiente e Desenvolvimento (ECO-92) e também reforçado pelo “Objetivo de Desenvolvimento Sustentável” de 2015, levando em consideração uma das questões mais importantes para a manutenção da qualidade do meio ambiente, o manejo adequado dos resíduos. ${ }^{27-28}$

A extrapolação das prevalências para o âmbito populacional apresentadas no artigo, reforçam a magnitude do problema a partir de uma amostra populacional. Projetar os resultados tem o potencial de auxiliar na organização de políticas públicas integradas, de modo a proteger a vida humana e o meio ambiente. Por meio dessas informações, é possível pensar estratégias de educação em saúde no cuidado ao idoso com diabetes e mostra o grave problema de saúde pública que reflete no cuidado individual de cada paciente, levando a complicações que geram hospitalizações e exposições evitáveis.

Quanto a extrapolação referente ao descarte do material, os dados mostram que se projetados para toda a população idosa com DM de Bagé, o número absoluto de usuários que podem descartar da maneira inadequada é alto, que além das consequências ambientais, coloca em risco trabalhadores que manuseiam o lixo contaminado. Pensar de maneira ampliada facilita o olhar crítico para os resultados e causa maior impacto na disseminação dos dados, procurando desenvolver ações de promoção da saúde dos idosos, cuidadores e profissionais para o cuidado integral e melhora da saúde da população, do manuseio de material, de modo a prevenir riscos ambientais e contaminação de outras pessoas.

Nesse contexto, o grande foco é trabalhar com a educação em saúde voltada à maior autonomia dos usuários, integração com serviços públicos de saúde, prevenção de agravos, na busca de condições adequadas de vida, pois permite a responsabilização de cada um pela redução dos riscos individuais e coletivos.

É necessária a implementação de estratégias educacionais voltadas para as pessoas com diabetes e seus familiares, com o objetivo de propagar práticas de autocuidado, desenvolver 
15 | Krolow MR, Machado KP, Oliveira AT, Soares MU, Volz PM, Thumé E

habilidades, atitudes e comportamentos para o manejo e controle da doença, além de melhorar a qualidade de vida de pacientes e retardar complicações. ${ }^{28-29}$

Como pontos positivos, destaca-se o percentual encontrado para essa amostra representativa dos idosos e projetando para o total de idosos do município, sendo possível observar a magnitude do problema e obter um panorama da situação de reutilização e descarte desses materiais, fornecendo indicadores para avaliação da qualidade e segurança no uso da insulinoterapia, além do delineamento de base populacional e do rigor metodológico empregado no planejamento e coleta de dados.

Algumas limitações neste estudo devem ser ponderadas, como o "n” do desfecho, considerado pequeno para as análises de associações com as variáveis independentes. Outro fator limitante foi a baixa publicação de artigos sobre os temas comprometendo a discussão com artigos atuais.

\section{Conclusão}

Os resultados deste estudo, mostram que a qualidade e a segurança adequada em relação ao uso e descarte dos materiais perfurocortantes por insulinodependentes se apresentou comprometida no município de Bagé, com mais da metade dos idosos reutilizando e descartando o material de forma errada.

Esses itens são passíveis de adequação por meio de intervenções simples como a educação em saúde. Ressalta-se que os dados encontrados podem auxiliar na tomada de decisões da gestão para organização da rede de saúde e investimentos em ações efetivas. E auxiliar os profissionais na formulação do processo de trabalho, baseado em evidências para orientar a população sobre a utilização e o descarte correto dos materiais para os insulinodependentes. 


\section{Referências}

1. Sociedade Brasileira de Diabetes (SBD). Diretrizes Sociedade Brasileira de Diabetes 2019-2020 [Internet]. 2019 [acesso em 2020 nov 15]. Disponível em: https://www.diabetes.org.br/profissionais/images/DIRETRIZES-COMPLETA-2019-2020.pdf

2. Mendoza Romo MA, Padrón Salas A, Cossío Torres PE, Soria Orozco M. Prevalencia mundial de la diabetes mellitus tipo 2 y su relación con el índice de desarrollo humano. Rev Panam Salud Publica. 2017;41:1-6. doi: https://doi.org/10.26633/RPSP.2017.103

3. Ministério da Saúde (BR). Diabetes: tipos, causas, sintomas, tratamento, diagnóstico e prevenção [Internet]. 2019 [acesso em 2019 jun 02]. Disponível em: http://www.saude.gov.br/saude-de-a-z/diabetes

4. Ministério da Saúde (BR). Estratégias para o cuidado da pessoa com doença crônica: Diabetes Mellitus [Internet]. Brasília (DF): Ministério da Saúde; 2013 [acesso em 2019 jun 30]. (Cadernos de Atenção Básica; 36).

Disponível em: https://bvsms.saude.gov.br/bvs/publicacoes/estrategias_cuidado_pessoa_diabetes_mellitus_cab36.pdf

5. Oliveira RG. Blackbook Enfermagem. Belo Horizonte: Blackbook; 2016.

6. Sociedade Brasileira de Diabetes (SBD). Posicionamento oficial SBD no 01/2017: recomendações sobre o tratamento injetável do Diabetes: insulinas e incretinas [Internet]. São Paulo: Sociedade Brasileira de Diabetes; 2017 [acesso em 2019 jun 10]. Disponível em: https://www.diabetes.org.br/profissionais/images/2017/posicionamento-oficial-sbd-01-2017.pdf

7. Bahendeka S, Kaushik R, Swai AB, Otieno F, Bajaj S, Kalra S, et al. EADSG Guidelines: insulin storage and optimisation of injection technique in Diabetes management. Diabetes Ther. 2019;10: 341-66. doi: https://doi.org/10.1007/s13300-019-0574-x

8. Secretaria Municipal de Saúde e Atenção à Pessoa com Deficiência do Município de Bagé. Plano municipal de saúde 2018-2021. Bagé: Secretaria Municipal de Saúde e Atenção à Pessoa com Deficiência; 2019 [acesso em 2020 jun 01]. Disponível em: https://www.bage.rs.gov.br/wpcontent/uploads/2019/02/PMS_2018_2021_Oficial_11022019.pdf

9. Ministério da Saúde (BR), Secretaria de Atenção Primária à Saúde (SAPS). e-Gestor Atenção Básica: informação da Atenção Básica. Brasília (DF): SAPS; 2017 [acesso em 2020 nov 18]. Disponível em: https://egestorab.saude.gov.br/paginas/acessoPublico/relatorios/relHistoricoCoberturaAB.xhtml

10. Thumé E, Facchini LA, Wyshak G, Campbell P. The utilization of home care by the elderly in Brazil's Primary Health Care System. Am J Public Health Res. 2011;101(5):868-74. doi: https://doi.org/10.2105/AJPH.2009.184648

11. BRASIL. Agência Nacional de Vigilância Sanitária (Brasil). Resolução RE no 2.605, de 11 de agosto de 2006. Estabelece a lista de produtos médicos enquadrados como de uso único proibidos de ser reprocessados. 
17 | Krolow MR, Machado KP, Oliveira AT, Soares MU, Volz PM, Thumé E

Diário Oficial da União: seção 1, Brasília, DF, n. 155, p. 37-38, 14 ago. 2006. Disponível em: http://bvsms.saude.gov.br/bvs/saudelegis/anvisa/2006/res2605_11_08_2006.html. Acesso em: 04 jun. 2019.

12. BRASIL. Agência Nacional de Vigilância Sanitária. Resolução RDC № 306, de 7 de dezembro de 2004. Dispõe sobre o Regulamento Técnico para o gerenciamento de resíduos de serviços de saúde. Diário Oficial da União: seção 1, Brasília, DF, n. 237, p. 49-56, 10 dez. 2004. Disponível em: https://bvsms.saude.gov.br/bvs/saudelegis/anvisa/2004/res0306_07_12_2004.html. Acesso em: 04 jun. 2014.

13. Associação Brasileira de Empresas de Pesquisa (ABEP). Critério Brasil 2020. São Paulo: ABEP; 2020 [acesso em 2020 nov 13]. Disponível em: http://www.abep.org/criterio-brasil

14. Fundação de Economia e Estatística (FEE). Estimativas populacionais (Revisão 2018). Porto Alegre: FEE; 2020 [acesso em 2020 maio 25]. Disponível em: https://arquivofee.rs.gov.br/indicadores/populacao/estimativas-populacionais-revisao-2018/

15. Iser BPM, Stopa SR, Chueiri PS, Szwarcwald CL, Malta DC, Monteiro HOC, et al. Prevalência de diabetes autorreferido no Brasil: resultados da Pesquisa Nacional de Saúde 2013. Epidemiol Serv Saúde. 2015;24(2):305-14. doi: https://doi.org/10.5123/S1679-49742015000200013

16. Francisco PMSB, Belon AP, Barros MBA, Carandina L, Alves MCGP, Goldbaun M, et al. Diabetes auto-referido em idosos: prevalência, fatores associados e práticas de controle. Cad Saúde Pública. 2010;26(1):175-84. doi: https://doi.org/10.1590/S0102-311X2010000100018

17. Vitoi NC, Fogal AS, Nascimento CM, Franceschini SCC, Ribeiro AQ. Prevalência e fatores associados ao diabetes em idosos no município de Viçosa, Minas Gerais. Rev Bras Epidemiol. 2015; 18(4):953-65. doi: https://doi.org/10.1590/1980-5497201500040022

18. Moreira APL, Malta DC, Vianna RPT, Moreira PVL, Carvalho AT. Risk and protection factors for self-reported hypertension and diabetes in João Pessoa, Brazil. The VIGITEL survey, 2014: a crosssectional study. São Paulo Med J. 2017;135(5):450-61. doi: https://doi.org/10.1590/15163180.2017.0044250517

19. Flores TR, Nunes BP, Assunção MCF, Bertoldi AD. Hábitos saudáveis: que tipo de orientação a população idosa está recebendo dos profissionais de saúde? Rev Bras Epidemiol. 2016;19(1): 167-80. doi: https://doi.org/10.1590/1980-5497201600010015

20. Francisco PMSB, Rodrigues PS, Costa KS, Tavares NUL, Tierling VL, Barros MBA, et al. Prevalência de diabetes em adultos e idosos, uso de medicamentos e fontes de obtenção: uma análise comparativa de 2012 e 2016. Rev Bras Epidemiol. 2019;22:e190061. doi: https://doi.org/10.1590/1980-549720190061

21. Dunes CAT, Sousa JK, Oliveira MTA. Reutilização de perfurocortantes na insulinoterapia e sua associação com infecções estafilocóccicas. Rev Epidemiol Controle Infecç. 2019;9(1):15-20. doi: https://doi.org/10.17058/reci.v9i1.12243 
22. Reis P, Marcon SS, Nass EMA, Arruda GO, Back IR, Lino IGT, et al. Desempenho de pessoas com diabetes mellitus na insulinoterapia. Cogitare Enferm. 2020;25. doi: https://doi.org/10.5380/ce.v25i0.66006

23. World Health Organization (WHO). Saving lives, spending less: a strategic response to noncommunicable diseases. Geneva: WHO; 2018 [cited 2019 Jun 02]. Available from: https://apps.who.int/iris/bitstream/handle/10665/272534/WHO-NMH-NVI-18.8-eng.pdf

24. Tapia CEV. Diabetes mellitus e o descarte de seringas e agulhas. Rev Gaúcha Enferm. 2009 [acesso em 2019 jun 09];30(2):228-34. Disponível em: https://www.seer.ufrgs.br/RevistaGauchadeEnfermagem/article/view/11310

25. Cunha GH, Barbosa RVA, Fontenele MSM, Lima MAC, Franco KB, Fechine FV. Insulin therapy waste produced in the households of people with diabetes monitored in Primary Care. Rev Bras Enferm. 2017;70(3):618-25. doi: https://doi.org/10.1590/0034-7167-2016-0406

26. André SCS, Veiga TB, Takayanagui AMM. Geração de resíduos de serviços de saúde em hospitais do município de Ribeirão Preto (SP), Brasil. Eng Sanit Ambient. 2016;21(1):123-30. doi: https://doi.org/10.1590/S1413-41520201600100140092

27. Organização das Nações Unidas Brasil. Objetivos do desenvolvimento sustentável: as Nações Unidas no Brasil. Brasília (DF): Organização das Nações Unidas; 2020 [acesso em 2020 nov 15]. Disponível em: https://brasil.un.org/pt-br/sdgs

28. Signor F, Leguisamo CP, De Marchi ACB, Bavaresco SS, Oliveira LZ, Pillatt AP. Opinião: conhecimento e educação em saúde de idosos portadores de diabetes mellitus. Fisioter Bras. 2016 [acesso 2020 jul 12];17(2):171-5. Disponível em: http://docs.bvsalud.org/biblioref/2018/02/879020/conhecimento-eeducacao-em-saude-de-idosos-portadores-de-diabet_pqiisIR.pdf

29. Gomes TF, Silva APL, Pereira BA, Rabelo NNF. Desenvolvimento de um programa de educação em Diabetes como ferramenta para a promoção da mudança de hábitos de vida: relato de experiência. Rev Atenas Higéia. 2019 [acesso 2019 jun 16];1(1):31-4. Disponível em: http://www.atenas.edu.br/revista/index.php/higeia/article/view/14

Editora Científica: Tânia Solange Bosi de Souza Magnago

Editora Associada: Carine Vendruscolo

\section{Autor correspondente}

Michele Rohde Krolow

E-mail: michele-mrk@hotmail.com

Endereço: Dr. José Alvares de Souza Soares Sobrinho 304

CEP: 96040460 
19 | Krolow MR, Machado KP, Oliveira AT, Soares MU, Volz PM, Thumé E

\section{Contribuições de Autoria}

1 - Michele Rohde Krolow

Concepção ou desenho do estudo/pesquisa, análise e/ou interpretação dos dados, revisão final com participação crítica e intelectual no manuscrito.

\section{2 - Karla Pereira Machado}

Concepção ou desenho do estudo/pesquisa, análise e/ou interpretação dos dados, revisão final com participação crítica e intelectual no manuscrito.

\section{3 - Adriéli Timm Oliveira}

Análise e/ou interpretação dos dados, revisão final com participação crítica e intelectual no manuscrito.

\section{4 - Mariangela Uhlmann Soares}

Análise e/ou interpretação dos dados, revisão final com participação crítica e intelectual no manuscrito.

\section{5 - Pâmela Moraes Volz}

Análise e/ou interpretação dos dados, revisão final com participação crítica e intelectual no manuscrito.

\section{6 - Elaine Thumé}

Concepção ou desenho do estudo/pesquisa, análise e/ou interpretação dos dados, revisão final com participação crítica e intelectual no manuscrito.

\section{Como citar este artigo}

Krolow MR, Machado KP, Oliveira AT, Soares MU, Volz PM, Thumé E. Qualidade e segurança no uso da insulinoterapia pela população idosa. Rev. Enferm. UFSM. 2021 [Acesso em: Ano Mês Dia]; vol.11 e38: 1-19. DOI:https://doi.org/10.5902/2179769248098 